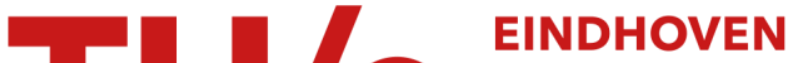 \\ UNIVERSITY OF \\ TECHNOLOGY
}

\section{Improving intersample behavior in discrete-time system inversion}

Citation for published version (APA):

van Zundert, J. C. D., Ohnishi, W., Fujimoto, H., \& Oomen, T. (2020). Improving intersample behavior in discrete-time system inversion: with application to LTI and LPTV systems. IEEE/ASME Transactions on Mechatronics, 25(1), 55-65. [8902055]. https://doi.org/10.1109/TMECH.2019.2953829

DOI:

10.1109/TMECH.2019.2953829

Document status and date:

Published: 01/02/2020

Document Version:

Accepted manuscript including changes made at the peer-review stage

Please check the document version of this publication:

- A submitted manuscript is the version of the article upon submission and before peer-review. There can be important differences between the submitted version and the official published version of record. People interested in the research are advised to contact the author for the final version of the publication, or visit the $\mathrm{DOI}$ to the publisher's website.

- The final author version and the galley proof are versions of the publication after peer review.

- The final published version features the final layout of the paper including the volume, issue and page numbers.

Link to publication

\section{General rights}

Copyright and moral rights for the publications made accessible in the public portal are retained by the authors and/or other copyright owners and it is a condition of accessing publications that users recognise and abide by the legal requirements associated with these rights.

- Users may download and print one copy of any publication from the public portal for the purpose of private study or research.

- You may not further distribute the material or use it for any profit-making activity or commercial gain

- You may freely distribute the URL identifying the publication in the public portal.

If the publication is distributed under the terms of Article $25 \mathrm{fa}$ of the Dutch Copyright Act, indicated by the "Taverne" license above, please follow below link for the End User Agreement:

www.tue.nl/taverne

Take down policy

If you believe that this document breaches copyright please contact us at:

openaccess@tue.nl

providing details and we will investigate your claim. 


\title{
Improving Intersample Behavior in Discrete-Time System Inversion: With Application to LTI and LPTV Systems
}

\author{
Jurgen van Zundert, Wataru Ohnishi, Member, IEEE, Hiroshi Fujimoto, Senior Member, IEEE, Tom Oomen, \\ Senior Member, IEEE
}

\begin{abstract}
Discrete-time system inversion for perfect tracking goes at the expense of intersample behavior. The aim of this paper is the development of a discrete-time inversion approach that improves continuous-time performance by also addressing the intersample behavior. The approach balances the on-sample and intersample behavior and provides a whole range of new solutions, with stable inversion and multirate inversion as special cases. The approach is successfully applied to an LPTV system in both simulations and experiments. The approach improves the intersample behavior through discrete-time system inversion and outperforms existing approaches.
\end{abstract}

Index Terms-Intersample behavior, discrete-time inversion, linear time invariant (LTI), linear periodically time varying (LPTV), stable inversion, multirate inversion

\section{INTRODUCTION}

Tracking control finds application in many areas, such as atomic force microscopes (AFMs) [1], wafer stages [2], and spectrometers [3]. The physical systems evolve in continuous time and hence their performance is naturally defined in continuous time. Many approaches for tracking control, including inverse model feedforward and iterative learning control (ILC), are based on system inversion. For continuous-time systems, system inversion approaches such as, for example, [4] can be used. However, controllers are often implemented in a digital environment since this provides a large flexibility at a low cost [5]. Due to the digital implementation, discrete-time control is often used.

One of the main challenges in system inversion is nonminimum-phase behavior. Causal inversion of nonminimum-phase systems yields unbounded inputs. To avoid unbounded inputs, many discrete-time inversion approaches have been proposed, see, e.g., [6] for a recent overview. Approximate inversion approaches such as zero phase error tracking control (ZPETC) [7], zero magnitude error tracking control (ZMETC), and nonminimum-phase zero-ignore (NPZ-Ignore) [8] are well-known, but yield limited performance since an approximation is used. Optimal

This work is part of the research programmes Robust Cyber-Physical Systems (RCPS) (No. 12694) and VIDI (No. 15698) which are both (partly) financed by the Netherlands Organisation for Scientific Research (NWO). The work is supported by JSPS KAKENHI Grant Number 18H05902.

Van Zundert and Oomen are with the Eindhoven University of Technology, Department of Mechanical Engineering, Control Systems Technology group, The Netherlands (e-mail: j.c.d.v.zundert@tue.nl, t.a.e.oomen@tue.nl).

Ohnishi and Fujimoto are with the University of Tokyo, Kashiwanoha, Kashiwa, Chiba, Japan (e-mail: ohnishi@ieee.org, fujimoto@k.u-tokyo.ac.jp). approaches such as norm-optimal feedforward, $\mathcal{H}_{2}$-preview control, and $\mathcal{H}_{\infty}$-preview control [6, Sections 4.3 and 4.4] yield high performance in discrete time. Discrete-time stable inversion [6, Section 4.2] yields exact tracking at the discrete-time samples.

Typically, discrete-time inversion approaches focus on the on-sample performance, i.e., at the discrete-time samples, resulting in poor intersample behavior, i.e., in between the samples, especially for zeros close to $z=-1$ [9]. This is observed for both linear time-invariant (LTI) and linear periodically time-varying (LPTV) systems [10]. As a consequence, the continuous-time behavior is poor. Indeed, the best onsample performance does not necessarily leads to the best continuous-time performance.

Multirate inversion [11], [12] provides an interesting alternative to improve intersample behavior by sacrificing onsample performance. However, the approach does not take into account the system dynamics when balancing the intersample and on-sample performance. As a consequence, the continuous-time performance will in general be sub-optimal.

Although there exist many discrete-time inversion approaches, the balance between on-sample performance and intersample behavior is often not addressed. The main contribution of this paper is a discrete-time inversion approach that finds the optimal balance between on-sample performance and intersample behavior for the purpose of continuous-time performance, for both LTI and LPTV systems. LPTV systems are of interest since they occur frequently, including sampled-data systems [5]; multirate systems [5], [13]; position-dependent systems with periodic tasks [14]; and non-equidistant sampling [15]. For both LTI and LPTV systems, the stable inversion and multirate inversion approaches are recovered as special cases. Related work includes [5], [16], [17] where synthesisbased approaches are presented. The approach presented in this paper does not require synthesis.

The outline of this paper is as follows. In Section II, the control diagram is presented and the control objective is formulated. The main idea of the approach and preliminary results are presented in Section III. The approach is presented in Section IV. The advantages of the approach are demonstrated by application to an LPTV motion system in simulations and experiments in Section V. Conclusions are presented in Section VI.

Notation. For notational convenience, single-input, singleoutput (SISO) systems are considered. The results can di- 


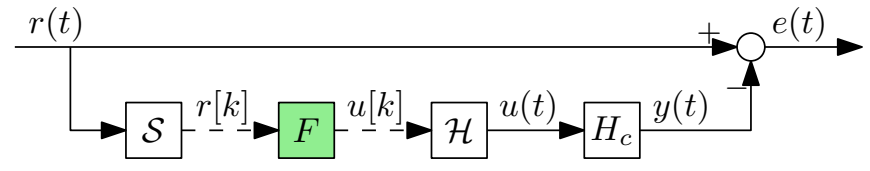

Fig. 1. Tracking control diagram with continuous-time system $H_{c}$, sampler $\mathcal{S}$, and hold $\mathcal{H}$. Given continuous-time reference trajectory $r(t)$, the objective is to minimize continuous-time error $e(t)$ through design of discrete-time controller $F$, while control input $u[k]$ remains bounded.

rectly be generalized to square multivariable systems. Let $s^{(i)} \triangleq \frac{\mathrm{d}^{i}}{\mathrm{~d} t^{i}} s$ denote the $i$ th time-derivative of $s, \mathcal{B}(\cdot)$ a bilinear transformation, and $\mathbb{R}_{>a}^{b}=\left\{x \in \mathbb{R}^{b} \mid x[k]>a\right.$ for all $k=$ $0,1, \ldots, b-1\}$. Let $\Sigma \stackrel{z}{=}(A, B, C, D)$ be a discrete-time statespace model and define the state transformation $\mathcal{T}(\Sigma, T) \stackrel{z}{=}$ $\left(T A T^{-1}, T B, C T^{-1}, D\right)$.

\section{PROBLEM FORMULATION}

In this section, the control problem is formulated. The considered tracking control configuration is shown in Fig. 1, with reference trajectory $r(t) \in \mathbb{R}$, control input $u(t) \in \mathbb{R}$, output $y(t) \in \mathbb{R}$, digital controller $F$, sampler $\mathcal{S}$, and zeroorder hold $\mathcal{H}$. The continuous-time, linear time-invariant (LTI) system $H_{c}$ is given by the minimal realization

$$
\begin{aligned}
\dot{x}(t) & =A_{c} x(t)+B_{c} u(t), \\
y(t) & =C_{c} x(t),
\end{aligned}
$$

with $x(t) \in \mathbb{R}^{n}, n \in \mathbb{N}$ and can be either an open-loop or closed-loop system. It is assumed that $H_{c}$ is stable.

In conventional discrete-time control, the focus is on onsample performance. The discrete-time system $H=\mathcal{S} H_{c} \mathcal{H}$ with $H_{c}$ in (1) and sampling time $\delta$ is given by

$$
\begin{aligned}
x[k+1] & =A x[k]+B u[k], \\
y[k] & =C x[k],
\end{aligned}
$$

with

$$
A=e^{A_{c} \delta}, \quad B=\int_{0}^{\delta} e^{A_{c} \tau} B_{c} \mathrm{~d} \tau, \quad C=C_{c} .
$$

In this setting, perfect on-sample tracking, i.e., $e[k]=0$, for all $k$, is achieved for $F=H^{-1}$. However, this does not provide any guarantees for the intersample performance $e(t), t \neq k \delta$. Hence, the continuous-time performance in terms of $e(t)$, for all $t$, may be poor as observed in, e.g., [10].

The control objective considered in this paper is to minimize the continuous-time error $e(t)$. Note that this includes both on-sample $(t=k \delta)$ and intersample $(t \neq k \delta)$ performance. Importantly, $u[k]$ should remain bounded, even in the presence of nonminimum-phase behavior. Trajectory $r(t)$ is assumed to be known a priori.

In the next section, the main idea of the approach and preliminary results are presented.

\section{CONCEPTUAL IDEA AND PRELIMINARY RESULTS}

In this section, the conceptual idea of the approach and preliminary results are presented. The results form the basis for the complete approach presented in Section IV.

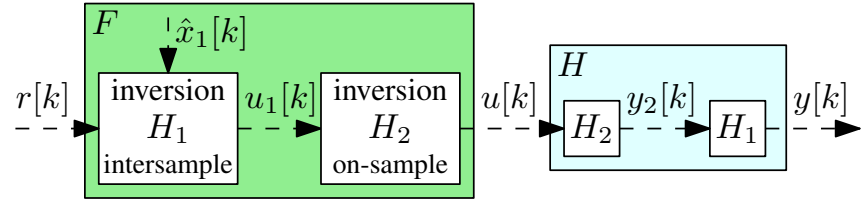

Fig. 2. Block diagram of the approach. The discrete-time system $H$ is decomposed into $H_{1}$ and $H_{2}$. System $H_{1}$ is inverted such that there is exact state tracking of the desired state $\hat{x}_{1}[k]$ every $n_{1}$ samples for the purpose of intersample behavior. System $\mathrm{H}_{2}$ is inverted such that there is exact output tracking every sample for the purpose of on-sample behavior.

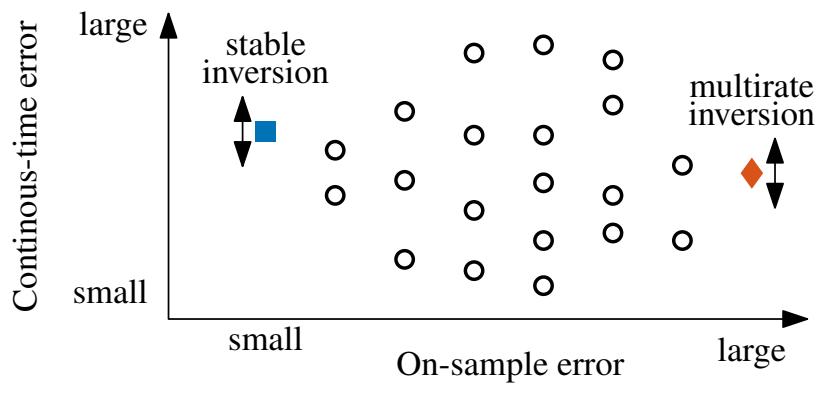

Fig. 3. Qualitative plot of the continuous-time versus on-sample error. The approach balances the intersample behavior and the on-sample behavior for the purpose of continuous-time performance. It provides a whole range of solutions $(\mathbf{O})$ that were non-existing before. Importantly, the smallest onsample error does not necessarily yield the smallest continuous-time error. The relative performance depends on the particular settings, e.g., the system dynamics, and may vary. Stable inversion $(\square)$ and multirate inversion $(\nabla)$ are recovered as special cases.

\section{A. Conceptual idea}

In the proposed approach, the system is decomposed into two parts and both parts are inverted separately according to Fig. 2, where $H$ is decomposed as $H=H_{1} H_{2}$. The inversion of system $H_{1}$ aims at the intersample behavior. More specific, let $n_{1}$ be the state dimension of $H_{1}$, then $H_{1}$ is inverted such that there is exact state tracking of a desired state $\hat{x}_{1}[k]$ every $n_{1}$ samples. The inversion of $H_{2}$ aims at the on-sample behavior through perfect output tracking for every sample.

Exact state tracking is experienced to yield good intersample behavior in multirate inversion [11], whereas exact output tracking yields good on-sample behavior in stable inversion [6]. Hence, the choice of the decomposition into $H_{1}$ and $\mathrm{H}_{2}$ can be used to balance the on-sample behavior and the intersample behavior to the benefit of the continuous-time performance. The idea is conceptually illustrated in Fig. 3. An important observation is that a small on-sample error does not necessarily yield a small continuous-time error. The figure shows that the approach provides a whole range of solutions that were non-existing before. The stable inversion and multirate inversion solution are recovered as the two extreme cases, see also Section IV-C.

The approach requires the decomposition $H=H_{1} H_{2}$ in terms of state-space realizations and the desired state $\hat{x}_{1}[k]$ for $H_{1}$, see also Fig. 2. In Section III-B, the desired state for the continuous-time system $H_{c}$ is presented. In Section III-C, the state-space decomposition $H=H_{1} H_{2}$ is presented. The results form the basis for the complete approach presented in Section IV. 


\section{B. Desired state for continuous-time system}

In this section, the desired state for the continuous-time system is presented. Given a continuous-time reference trajectory $r(t)$ together with its $n-1$ time derivatives and system $H_{c}$ in (1), the objective is to determine a bounded state $\hat{x}(t)$ such that $y(t)=C_{c} \hat{x}(t)$ yields $y^{(i)}(t)=r^{(i)}(t), i=0,1, \ldots, n-1$, where $(\cdot)^{(i)}$ denotes the $i$ th time derivative of $(\cdot)$, i.e., such that $\bar{r}(t)=\bar{y}(t)$ where

$$
\bar{r}(t)=\left[\begin{array}{c}
r^{(0)}(t) \\
r^{(1)}(t) \\
\vdots \\
r^{(n-1)}(t)
\end{array}\right], \quad \bar{y}(t)=\left[\begin{array}{c}
y^{(0)}(t) \\
y^{(1)}(t) \\
\vdots \\
y^{(n-1)}(t)
\end{array}\right] .
$$

A similar approach as in [11] is used based on the controllable canonical form given by Lemma 1 , see also [18, Section 17.6]. The desired state is given by Theorem 2. The results are recapitulated for completeness and reading convenience.

Lemma 1 (Controllable canonical form). Let the transfer function of $H_{c}$ in (1) be given by

$$
H_{c}=C_{c}\left(s I-A_{c}\right)^{-1} B_{c}=\frac{B(s)}{A(s)},
$$

with

$$
\begin{aligned}
& A(s)=\frac{s^{n}+a_{n-1} s^{n-1}+\ldots+a_{0}}{b_{0}}, \\
& B(s)=\frac{b_{m} s^{m}+b_{m-1} s^{m-1}+\ldots+b_{0}}{b_{0}},
\end{aligned}
$$

$b_{0} \neq 0$, then the controllable canonical form $H_{c c f}=$ $\mathcal{T}\left(H_{c}, T_{c c f}\right)$ is given by

$$
\begin{aligned}
\dot{x}_{c c f}(t) & =A_{c c f} x_{c c f}(t)+B_{c c f} u(t), \\
y(t) & =C_{c c f} x_{c c f}(t),
\end{aligned}
$$

where

$$
\left[\begin{array}{l|l}
A_{c c f} & B_{c c f} \\
\hline C_{c c f} &
\end{array}\right]=\left[\begin{array}{ccccc|c}
0 & 1 & 0 & \cdots & 0 & 0 \\
0 & 0 & 1 & \cdots & 0 & 0 \\
\vdots & \vdots & \vdots & \ddots & \vdots & \vdots \\
0 & 0 & 0 & \cdots & 1 & 0 \\
-a_{0} & -a_{1} & -a_{2} & \cdots & -a_{n-1} & b_{0} \\
\hline 1 & \frac{b_{1}}{b_{0}} & \frac{b_{2}}{b_{0}} & \cdots & 0 &
\end{array}\right]
$$

and

$$
T_{c c f}^{-1}=\left[\begin{array}{llll}
B_{c} & A_{c} B_{c} & \cdots & A_{c}^{n-1} B_{c}
\end{array}\right]\left[\begin{array}{cccc}
\frac{a_{1}}{b_{0}} & \frac{a_{2}}{b_{0}} & \cdots & \frac{1}{b_{0}} \\
\frac{a_{2}}{b_{0}} & \frac{a_{3}}{b_{0}} & \cdot & \cdot \\
\vdots & \cdot & 0 \\
\frac{1}{b_{0}} & 0 & \cdots & 0
\end{array}\right] .
$$

Theorem 2 (Desired continuous-time state). Let $B^{-1}(s)$ in (4) be decomposed as

$$
B^{-1}(s)=F_{s}(s)+F_{u}(s)
$$

with all poles $p_{s} \in \mathbb{C}$ of $F_{s}(s)$ such that $\Re\left(p_{s}\right)<0$ and all poles $p_{u} \in \mathbb{C}$ of $F_{u}(s)$ such that $\Re\left(p_{u}\right)>0$. Let

$$
\begin{aligned}
f_{s}(t) & =\mathcal{L}^{-1}\left(F_{s}(s)\right), \quad f_{u}(t)=\mathcal{L}^{-1}\left(F_{u}(-s)\right), \\
\hat{x}_{c c f, s}(t) & =\int_{-\infty}^{t} f_{s}(t-\tau) \bar{r}(\tau) d \tau, \\
\hat{x}_{c c f, u}(t) & =\int_{t}^{\infty} f_{u}(t-\tau) \bar{r}(\tau) d \tau,
\end{aligned}
$$

where $\mathcal{L}^{-1}(\cdot)$ is the inverse uni-lateral Laplace transform [19, Section 9.3]. Let $H_{c}$ in (4) have realization (5), then $y(t)=$ $C_{c} \hat{x}(t)$ where

$$
\hat{x}(t)=T_{c c f}^{-1}\left(\hat{x}_{c c f, s}(t)+\hat{x}_{c c f, u}(t)\right),
$$

is bounded and such that $\bar{y}(t)=\bar{r}(t)$, with $\bar{y}(t), \bar{r}(t)$ in (3).

Proof: See [11, Section IV.B].

Theorem 2 provides the desired bounded state for optimal state tracking. Together with the state-space decomposition presented in the next section, Theorem 2 forms the basis of the approach presented in Section IV.

Remark 1. If poles of $B^{-1}(s)$ in Theorem 2 have $\Re(p)=0$, i.e., $B^{-1}(s)$ is non-hyperbolic, similar techniques as in [20] can be used.

\section{State-space decomposition}

In this section, the multiplicative state-space decomposition is presented. A multiplicative decomposition, in contrast to an additive decomposition, enables exact on-sample tracking every few samples. Together with Theorem 2, the decomposition forms the basis of the approach in Section IV.

Given the state-space system $H$ in (2), the interest is in minimal realizations $H_{1}, H_{2}$ such that $H=H_{1} H_{2}$ in terms of state-space realization, where the zeros and poles of $H$ can be arbitrarily assigned to $H_{1}$ or $H_{2}$. The starting point is the multiplicative decomposition $H=H_{1} H_{2}$ in terms of transfer functions as given by Lemma 3.

Lemma 3 (Transfer function decomposition). Let $H \stackrel{z}{=}$ $(A, B, C, D)$ be a state-space realization with $n$ states and invertible $D$. Let $V \in \mathbb{R}^{n \times n_{1}}$ be a column space of an invariant subspace of $A$ and let $V_{\times} \in \mathbb{R}^{n \times n_{2}}$ be a column space of an invariant subspace of $A_{\times}=A-B D^{-1} C$, such that $S=\left[\begin{array}{ll}V & V_{\times}\end{array}\right]$has full rank $n=n_{1}+n_{2}$. Let

$$
\Pi=S\left[\begin{array}{cc}
I_{n_{1}} & 0_{n_{1} \times n_{2}} \\
0_{n_{2} \times n_{1}} & 0_{n_{2} \times n_{2}}
\end{array}\right] S^{-1} .
$$

Then, the realizations

$$
H_{1 f} \stackrel{z}{=}\left[\begin{array}{c|c}
A & \Pi B D^{-1} \\
\hline C & I
\end{array}\right], H_{2 f} \stackrel{z}{=}\left[\begin{array}{c|c}
A & B \\
\hline C(I-\Pi) & D
\end{array}\right]
$$

are such that $H=H_{1 f} H_{2 f}$ in terms of transfer functions, i.e., $C(z I-A)^{-1} B+D=\left(C(z I-A)^{-1} \Pi B D^{-1}+I\right)(C(I-$ ПI) $\left.(z I-A)^{-1} B+D\right)$.

Proof: Follows directly from extending [21, Corrollary 11] to $D \neq I$.

If the $D$ matrix in Lemma 3 is singular, a bilinear transformation [5, Section 3.4]; [19, Section 10.8.3] can possibly be employed to obtain an equivalent system with non-singular $D$ matrix. A multiplicative decomposition for the transformed system is obtained through Lemma 3. Applying the inverse transformation on the decomposed system yields the decomposition for the original system since $\mathcal{B}\left(H_{1} H_{2}\right)=\mathcal{B}\left(H_{1}\right) \mathcal{B}\left(H_{2}\right)$.

Importantly, Lemma 3 guarantees equivalence in terms of transfer functions, but not in terms of state-space realizations. Indeed, the decomposition of Lemma 3 yields nonminimal 
realizations of $H_{1 f}, H_{2 f}$ as both have state dimension $n$. By exploiting the modal form and using a suitable state transformation, the desired state-space decomposition for the approach is obtained as given by Theorem 4 .

Theorem 4 (State-space decomposition). Let $T_{\text {mod }} \in \mathbb{C}^{n \times n}$ be such that $H_{\text {mod }}=\mathcal{T}\left(H, T_{\text {mod }}\right)=(A, B, C, D)$ is in modal form [22, Section 7.4] with nonsingular D. Let $H_{1 f} H_{2 f}=$ $H_{\text {mod }}$ be the decomposition given by Lemma 3. Let $T_{p e r} \in$ $\mathbb{R}^{n \times n}$ be such that

$$
\begin{aligned}
& \mathcal{T}\left(H_{1 f}, T_{\text {per }}\right) \stackrel{z}{=}\left[\begin{array}{cc|c}
A_{1} & 0 & B_{1} \\
0 & A_{2} & 0 \\
\hline C_{1} & C_{1 r} & I
\end{array}\right], \\
& \mathcal{T}\left(H_{2 f}, T_{p e r}\right) \stackrel{z}{=}\left[\begin{array}{cc|c}
A_{1} & 0 & B_{2 r} \\
0 & A_{2} & B_{2} \\
\hline 0 & C_{2} & D
\end{array}\right],
\end{aligned}
$$

with $A_{1} \in \mathbb{R}^{n_{1} \times n_{1}}, A_{2} \in \mathbb{R}^{n_{2} \times n_{2}}, n_{1}+n_{2}=n$, and define

$$
H_{1} \stackrel{z}{=}\left[\begin{array}{c|c}
A_{1} & B_{1} \\
\hline C_{1} & I
\end{array}\right], \quad H_{2} \stackrel{z}{=}\left[\begin{array}{c|c}
A_{2} & B_{2} \\
\hline C_{2} & D
\end{array}\right] .
$$

Furthermore, let $X \in \mathbb{R}^{n_{1} \times n_{2}}$ satisfy

$$
A_{1} X-X A_{2}=B_{1} C_{2} .
$$

Then, the state-space realization of $\mathcal{T}\left(H_{1} H_{2}, T_{p e r}^{-1} T_{12}\right)$ with

$$
T_{12}=\left[\begin{array}{cc}
I_{n_{1}} & X \\
0_{n_{1} \times n_{2}} & I_{n_{2}}
\end{array}\right],
$$

is identical to that of $H_{\text {mod }}$.

Proof: See Appendix A.

Theorem 4 yields a state-space decomposition $H=H_{1} H_{2}$ with identical state-space realizations. Note that such a decomposition always exists. Together with Theorem 2, Theorem 4 forms the basis for the approach presented in the next section.

Remark 2. Note that $V$ in Lemma 3 is related to the poles of $H$, whereas $V_{\times}$is related to the zeros of $H$. Hence, $V, V_{\times}$ can be used to assign the poles and zeros to either $\mathrm{H}_{1}$ or $\mathrm{H}_{2}$.

Remark 3. The column spaces of the invariant subspaces in Lemma 3 can be constructed from eigenvectors. Note that for complex eigenvectors, the real and imaginary part should be used. For eigenvalues with multiplicity larger than 1, generalized eigenvectors obtained from the Jordan form can be used to ensure $S$ has full rank.

Remark 4. Sylvester equation (15) has a unique solution $X$ if the eigenvalues of $A_{1}$ and $-A_{2}$ are distinct [23].

\section{INVERSION FOR INTERSAMPLE PERFORMANCE}

In the previous section, the global idea and preliminary results on the desired state and the state-space decomposition are presented. Based on these results, the approach is presented. First, the approach for LTI systems is presented. Second, the approach for LPTV systems is presented. Finally, special cases are recovered.

\section{A. Approach for LTI systems}

The approach consists of two steps. First, stable inversion is applied to $H_{2}$ in (14) to obtain $u[k]$ such that $y_{2}[k]=u_{1}[k]$, for all $k$, see also Fig. 2. The solution is given by Theorem 5 and provides exact output tracking every sample. See $[6$, Section 4.2] for a proof.

Theorem 5 (Inversion of $\mathrm{H}_{2}$ ). Consider Fig. 2 and let $\mathrm{H}_{2}^{-1}$ be given by

$$
\begin{aligned}
{\left[\begin{array}{l}
x_{s}[k+1] \\
x_{u}[k+1]
\end{array}\right] } & =\left[\begin{array}{cc}
A_{s} & 0 \\
0 & A_{u}
\end{array}\right]\left[\begin{array}{l}
x_{s}[k] \\
x_{u}[k]
\end{array}\right]+\left[\begin{array}{l}
B_{s} \\
B_{u}
\end{array}\right] u_{1}[k], \\
u[k] & =\left[\begin{array}{ll}
C_{s} & C_{u}
\end{array}\right]\left[\begin{array}{l}
x_{s}[k] \\
x_{u}[k]
\end{array}\right]+D u_{1}[k],
\end{aligned}
$$

with $\left|\lambda\left(A_{s}\right)\right|<1$ and $\left|\lambda\left(A_{u}\right)\right|>1$. Then, $y_{2}[k]=u_{1}[k]$, for all $k$, if

$$
u[k]=C_{s} x_{s}[k]+C_{u} x_{u}[k]+D u_{1}[k],
$$

which is bounded for bounded $u_{1}$ and where $x_{s}$ follows from solving

$$
x_{s}[k+1]=A_{s} x_{s}[k]+B_{s} u_{1}[k], \quad x_{s}[-\infty]=0
$$

forward in time and $x_{u}$ follows from solving

$$
x_{u}[k+1]=A_{u} x_{u}[k]+B_{u} u_{1}[k], \quad x_{u}[\infty]=0
$$

backward in time.

If $u_{1}[k]$ is bounded, $u[k]$ in Theorem 5 is bounded by construction of $x_{s}[k], x_{u}[k]$, even if $H_{2}$ is nonminimum phase. The stable inversion solution in Theorem 5 achieves exact output tracking every sample and has infinite pre-actuation. Regular causal inversion is recovered as special case if the system is minimum phase ( $x_{u}$ is non-existing), see also [6].

Second, multirate inversion is applied to $H_{1}$ in (14) to obtain $u_{1}[k]$. Note that by Theorem $5, y_{2}[k]=u_{1}[k]$, for all $k$. The solution is based on lifting the state equation over $n_{1}$ samples. The solution is adopted from [11] and given by Theorem 6 . The solution provides exact state tracking every $n_{1}$ samples.

Theorem 6 (Inversion of $H_{1}$ ). Consider Fig. 2 with $y_{2}[k]=$ $u_{1}[k]$, for all $k$, and let $\hat{x}_{1}[k]$ be the desired state for system $H_{1}$ in (14). Consider the state equation lifted over $\tau$ samples given by

$$
\underline{x}_{1}[q+1]=\underline{A}_{1} \underline{x}_{1}[q]+\underline{B}_{1} \underline{u}_{1}[q],
$$

with $\underline{A}_{1}=A_{1}^{n_{1}}, \underline{B}_{1}=\left[\begin{array}{llll}A_{1}^{n_{1}-1} B_{1} & A_{1}^{n_{1}-2} B_{1} & \ldots & B_{1}\end{array}\right]$, $\underline{u}_{1}[q]=\left[\begin{array}{llll}u_{1}\left[k n_{1}\right] & u_{1}\left[k n_{1}+1\right] & \ldots & u_{1}\left[(k+1) n_{1}-1\right.\end{array}\right]^{\top}$, and $\underline{x}_{1}[q]=x_{1}\left[k n_{1}\right]$. Then, $\underline{x}_{1}[q]=\underline{\hat{x}}_{1}[q]$, for all $q$, where $\underline{\hat{x}}_{1}[q]$ is $\hat{x}_{1}[k]$ lifted over $\tau$ samples, if

$$
\underline{u}_{1}[q]=\underline{B}_{1}^{-1}\left(\underline{\hat{x}}_{1}[q+1]-\underline{A}_{1} \underline{\hat{x}}_{1}[q]\right),
$$

which is bounded for bounded $\hat{x}_{1}$.

Proof: See [11, Section IV.C].

Importantly, the inversion approach in Theorem 6 is based on the continuous-time system $H_{c}$, rather than the discretetime system $H$. The approach yields exact state tracking, and hence exact output tracking, every $n_{1}$ samples and has $n_{1}$ samples pre-actuation. Note that $u_{1}$ is bounded if $\hat{x}_{1}$ is 
bounded, even if $H_{1}$ is nonminimum phase. More details can be found in, for example, [11], [12]. The desired state $\hat{x}_{1}$ in Theorem 6 is obtained by Procedure 7 which follows from Section III-B and Section III-C. An example of the application of Procedure 7 to a motion system is provided in Appendix B.

Procedure 7 (Desired state of $H_{1}$ ). Given $H_{c}$ in (5), $H$ in (2), and the decomposition $H=H_{1} H_{2}$ in Theorem 4, the following steps yields the desired state $\hat{x}_{1}[k]$ in Theorem 6.

1) Obtain the controllable canonical form $H_{c c f}=$ $\mathcal{T}\left(H_{c}, T_{c c f}\right)$ according to Lemma 1.

2) Obtain the desired state $\hat{x}(t)$ of $H_{c}$ using Theorem 2.

3) Set the desired state of $H$ to $\hat{x}[k]=\hat{x}(k \delta)$.

4) Obtain the desired state of $H_{\text {mod }}: \hat{x}_{\text {mod }}[k]=T_{\text {mod }} \hat{x}[k]$, with $H_{\text {mod }}, T_{\text {mod }}$ in Theorem 4.

5) Given $H_{1}, H_{2}$ in (14), let

$$
H_{12}=H_{1} H_{2} \stackrel{z}{=}\left[\begin{array}{cc|c}
A_{1} & B_{1} C_{2} & B_{1} D_{2} \\
0 & A_{2} & B_{2} \\
\hline C_{1} & D_{1} C_{2} & D_{1} D_{2}
\end{array}\right]
$$

6) Obtain the desired state of $H_{12}: \hat{x}_{12}[k]=$ $T_{12}^{-1} T_{\text {per }} \hat{x}_{\text {mod }}[k]$, with $T_{12}$ in (16) and $T_{\text {per }}$ satisfying (12) and (13).

7) Obtain the desired state for $H_{1}: \hat{x}_{1}[k]=$ $\left[\begin{array}{ll}I_{n_{1}} & 0_{n_{1} \times n_{2}}\end{array}\right] \hat{x}_{12}[k]$.

The combination of the inversion of $\mathrm{H}_{2}$ in Theorem 5 and the inversion of $H_{1}$ in Theorem 6 constitutes the control input $u[k]$ in Fig. 2, which is bounded by design, also for nonminimum-phase systems. The design freedom is in the decomposition of $H$ into $H_{1}$ and $H_{2}$ in Theorem 4. Equation (23) shows that the output is given by $y[k]=C_{1} x_{1}[k]+D_{1} C_{2} x_{2}[k]$ since either $D_{1}=0$ or $D_{2}=0$ as $D=0$ in (2). If $D_{1}=0$, $y[k]=C_{1} x_{1}[k]$ and since inversion of $H_{1}$ in Theorem 5 ensures perfect state tracking of $x_{1}[k]$ every $n_{1}$ samples, there is perfect output tracking every $n_{1}$ samples. If $D_{1} \neq 0, y[k]$ also depends on $x_{2}[k]$ of $\mathrm{H}_{2}$ and since inversion of $\mathrm{H}_{2}$ in Theorem 6 does not provide perfect state tracking, there is no perfect output tracking for $y[k]$ every $n_{1}$ samples. Hence, to guarantee exact on-sample tracking every $n_{1}$ samples, $V, V_{\times}$ in Theorem 4 are preferably chosen such that $D_{1}=0$.

In this section, the approach for LTI systems is presented. In the next section, the approach for LPTV systems is presented.

Remark 5. For strictly proper systems $\mathrm{H}_{2}$, Theorem 5 can be applied to the bi-proper system $\bar{H}_{2}$ obtained through time shifts $\bar{H}_{2}=z^{d_{2}} H_{2}$, where $d_{2}$ is the relative degree of $\mathrm{H}_{2}$, see also [6, Remark 1]. If there are eigenvalues on the unit circle, i.e., there exist $\lambda_{i}$ such that $\left|\lambda_{i}(A)\right|=1$, then similar techniques as in [20] can be followed.

Remark 6. The decomposition of $H^{-1}$ given by (17) can be obtained through an eigenvalue decomposition.

Remark 7. Note that $\underline{B}_{1}$ in Theorem 6 is the controllability matrix of $H_{1}$ and hence $\underline{B}_{1}^{-1}$ exists if $H_{1}$ is controllable.

\section{B. Approach for LPTV systems}

In this section, the approach for LPTV systems is presented. Let the LPTV system $H$ with period $\tau \in \mathbb{N}$ be given by

$$
\begin{aligned}
x[k+1] & =A[k] x[k]+B[k] u[k], \\
y[k] & =C[k] x[k],
\end{aligned}
$$

with $A[k+\tau]=A[k], B[k+\tau]=B[k], C[k+\tau]=C[k]$, for all $k$. LPTV systems may result from non-equidistant sampling as in Example 1.

Example 1 (Non-equidistant sampling). Let the sampling in Fig. 1 be non-equidistant in time and given by the sampling sequence $\Delta_{n e} \in \mathbb{R}_{>0}^{\infty}$ with periodicity $\tau \in \mathbb{N}$ defined as

$$
\Delta_{n e}=\left(\delta_{1}, \delta_{2}, \ldots, \delta_{\tau}, \delta_{1}, \delta_{2} \ldots\right),
$$

with $\delta_{i}=\gamma_{i} \delta_{b}, \delta_{b} \in \mathbb{R}_{>0}, \gamma_{i} \in \mathbb{N}, i=1,2, \ldots, \tau$. Then, the discretized system $H=\mathcal{S} H_{c} \mathcal{H}$ is given by (24) with

$$
A[i]=e^{A_{c} \delta_{i}}, \quad B[i]=\int_{0}^{\delta_{i}} e^{A_{c} \tau} B_{c} d \tau, \quad C=C_{c},
$$

$i=1,2, \ldots, \tau$, where $A[k+\tau]=A[k], B[k+\tau]=B[k]$, for all $k$. By linearity of $H_{c}$ and periodicity of $\Delta_{n e}, H$ is LPTV with period $\tau$.

The approach for LPTV systems is similar to that for LTI systems, with the key difference that an additional lifting step is used. The lifting step turns the LPTV system into a (multivariable) LTI system as given by Lemma 8 .

Lemma 8. Lifting the input of $H$ in (24) over $\tau$ samples yields the LTI system $\underline{H}$ given by

$$
\begin{aligned}
\underline{x}[q+1] & =\underline{A} \underline{x}[q]+\underline{B} \underline{u}[q], \\
\underline{y}[q] & =\underline{C} \underline{x}[q]+\underline{D} \underline{u}[q],
\end{aligned}
$$

where

$$
\underline{x}[q]=x[k \tau], \quad \underline{u}[q]=\left[\begin{array}{c}
u[k \tau] \\
u[k \tau+1] \\
\vdots \\
u[(k+1) \tau-1]
\end{array}\right],
$$

$$
\begin{aligned}
& {\left[\begin{array}{c|c}
\underline{A} & \underline{B} \\
\hline \underline{C} & \underline{D}
\end{array}=\right.} \\
& {\left[\begin{array}{c|cccc}
\Phi_{\tau+1,1} & \Phi_{\tau+1,2} B[1] & \Phi_{\tau+1,3} B[2] & \ldots & B[\tau] \\
\hline C[1] & 0 & 0 & \cdots & 0 \\
C[2] \Phi_{2,1} & C[2] B[1] & 0 & \cdots & 0 \\
\vdots & \vdots & \vdots & \ddots & \vdots \\
C[\tau] \Phi_{\tau, 1} & C[\tau] \Phi_{\tau, 2} B[1] & C[\tau] \Phi_{\tau, 3} B[2] & \ldots & 0
\end{array}\right],}
\end{aligned}
$$

with transition matrix

$$
\Phi_{k_{2}, k_{1}}= \begin{cases}I, & k_{2}=k_{1}, \\ A\left[k_{2}-1\right] A\left[k_{2}-2\right] \ldots A\left[k_{1}\right], & k_{2}>k_{1} .\end{cases}
$$

For the lifted system $\underline{H}$ in (27), the same approach as for the LTI system illustrated in Fig. 2 is used. The state-space decomposition $\underline{H}=\underline{H}_{1} \underline{H}_{2}$ is obtained using Theorem 4 . System $\underline{H}_{2}$ is inverted using Theorem 5 and $\underline{H}_{1}$ is inverted using Theorem 6 , where the desired state $\underline{\hat{x}}_{1}[q]$ follows along the same lines as in Procedure 7. The result is the lifted input signal $\underline{u}[q]$, which, after inverse lifting, yields input $u[k]$, for the LPTV system $H$ in (24). 
In the previous and present section, the approaches for LTI and LPTV are presented, respectively. Next, special cases are recovered.

\section{Special cases}

The approach provides a whole range of solutions as illustrated in Fig. 3. The stable inversion and multirate inversion solution are recovered as the two extreme cases and given by Corollary 9 and Corollary 10. The results hold for both LTI and LPTV systems.

Corollary 9 (Special case: stable inversion [6], [24]). The stable inversion solution for $H$ is recovered from the approach in Section IV as special case if $H=H_{2}$, i.e., $H_{1}=I$ and $n_{1}=0$.

Corollary 10 (Special case: multirate inversion [11], [12]). The multirate inversion solution for $H$ is recovered from the approach in Section IV as special case if $H=H_{1}$, i.e., $H_{2}=I$ and $n_{1}=n$.

Importantly, although Theorem 5 yields exact output tracking of $H_{2}$ for every sample, the inversion of $H_{1}$ does not reduce to conventional multirate inversion of $H_{1}$ since the desired state $\hat{x}_{1}[k]$ depends on the full system $H_{c}$ and not only on $H_{1}$.

Finally, the approach for LTI systems is recovered from that for LPTV systems as given by Corollary 11. Indeed, for $\tau=1$, (2) is recovered from (24).

Corollary 11. The approach is applicable to both LTI and LPTV systems. Indeed, the approach for LTI systems in Section IV-A is recovered as a special case from the approach for LPTV systems in Section IV-B for $\tau=1$.

The approach provides a whole range of solutions that were non-existing before, see also Fig. 3. The advantages of the approach are demonstrated by application to an LPTV motion system in Section V.

\section{EXPERIMENTAL RESULTS}

In this section, the approach outlined in Section IV is validated experimentally on a motion system. The experiments show the applicability of the approach in a practical setting. First, the motion system is presented. Second, the approach is validated in simulations revealing the desired properties and providing the optimal decomposition for use in the experiments. Finally, the approach is validated in experiments. Both in simulations and in experiments the approach is superior to the special cases of stable inversion and multirate inversion.

\section{A. Motion system}

The approach is validated on the experimental highprecision positioning stage shown in Fig. 4(a). The Bode diagram of a frequency response function measurement of the system is shown in Fig. 4(b). The measurement is obtained through a dedicated identification experiment with a multisine

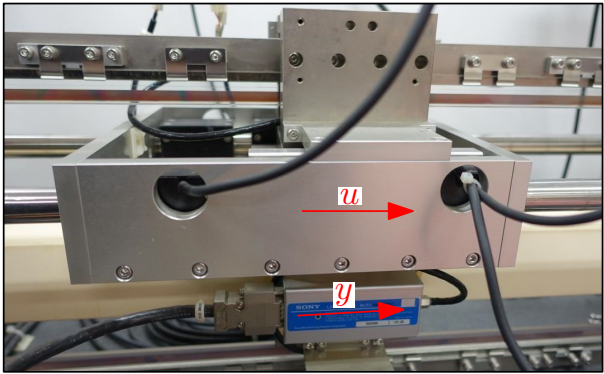

(a) Experimental high-precision positioning stage with force input $u$ and output displacement $y$.
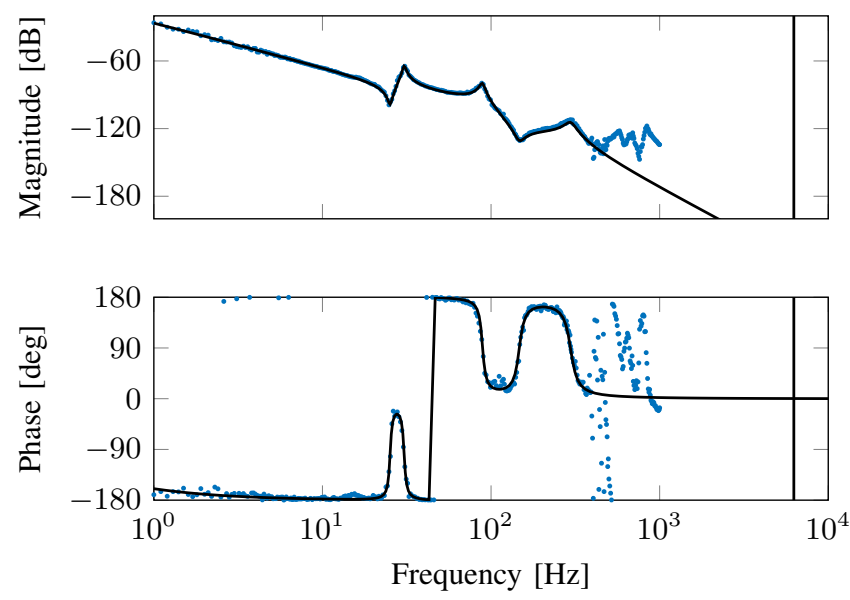

(b) Bode diagram of a frequency response function measurement $(\bullet)$ of the motion system in Fig. 4(a) and the identified continuous-time model $H_{c}$ $(-)$.

Fig. 4. Motion system [25] used in simulations and for experimental validation.

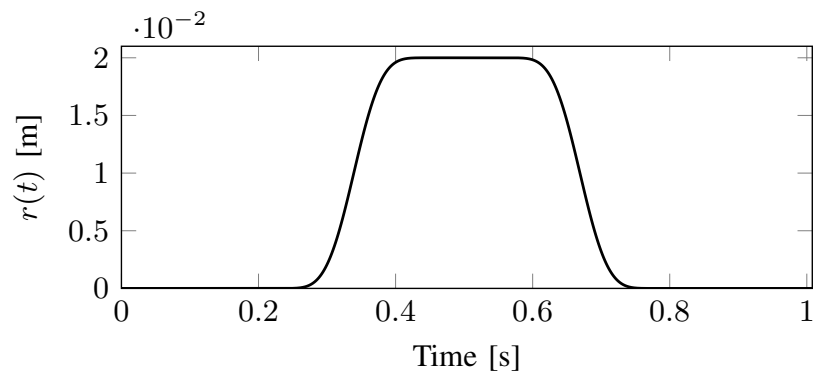

Fig. 5. Reference trajectory $r(t)$ consisting of 8th order polynomials in time.

input in the range between $1-1000 \mathrm{~Hz}$ and a sampling frequency of $12.5 \mathrm{kHz}$. The identified 8th order continuous-time system $H_{c}(n=8$ and $m=4)$ is given by

$$
\begin{aligned}
& H_{c}=\frac{3.7232 \cdot 10^{6}\left(s^{2}+7.181 s+2.507 \cdot 10^{4}\right)}{s(s+2.33)\left(s^{2}+9.132 s+3.672 \cdot 10^{4}\right)} \\
& \times \frac{\left(s^{2}+102.6 s+8.531 \cdot 10^{5}\right)}{\left(s^{2}+37.91 s+3.12 \cdot 10^{5}\right)\left(s^{2}+254.5 s+3.478 \cdot 10^{6}\right)}
\end{aligned}
$$

and is stable and minimum phase. The Bode diagram of the model $H_{c}$ is also shown in Fig. 4(b). The continuous-time reference trajectory $r(t)$ is shown in Fig. 5.

A non-equidistant sampling sequence with $\gamma_{1}=1, \gamma_{2}=2$, and $\delta_{b}=400 \mu \mathrm{s}\left(f_{b}=2.5 \mathrm{kHz}\right)$ is used, see also Example 1, resulting in an LPTV system $H$ with period $\tau=2$. The corre- 
sponding lifted LTI system $\underline{H}$ in (27) has one nonminimumphase (transmission) zero due to discretization.

\section{B. Simulation: finding the optimal decomposition}

In this section, the approach in Section IV is evaluated in simulation to confirm its properties and to find the optimal decomposition for use in the experiments. In Section V-C, the approach is validated in experiments.

First, three different solutions are considered: the approach in Section IV with $n_{1}=4$, the special case $n_{1}=0$, i.e., multirate inversion in Corollary 10, and the special case $n_{1}=8$, i.e., stable inversion in Corollary 9. The input signals are shown in Fig. 6. Note that the input signal of stable inversion is non smooth. The error signals are shown in Fig. 7 which, for the purpose of intersample performance evaluation, are evaluated at a sampling frequency of $250 \mathrm{kHz}$, i.e., a factor 100 higher than $f_{b}$.

The special case of stable inversion in Fig. 7(a) achieves perfect output tracking, however, the intersample performance is poor as a result of the non-smooth input signal, see Fig. 6 . The special case of multirate inversion in Fig. 7(b) yields perfect state tracking every $n=8$ samples, with reasonable intersample performance. The approach in Fig. 7(c) achieves perfect state tracking every $n_{1}=4$ samples and good intersample performance. The proposed approach outperforms the special cases of stable inversion and multirate inversion in terms of the continuous-time error $e(t)$.

Second, the performance is evaluated for a variety of solutions. The results are shown in Fig. 8 and quantify Fig. 3 . The results show that many of the solutions provided by the approach outperform the special cases of stable inversion and multirate inversion. Note that Fig. 8 only shows results for even numbers $n_{1}$ due to the additional lifting step with $\tau=2$ that is presently used. The solution shown in Fig. 6(c) and Fig. 7(c) is the solution which yields the best performance as indicated by in Fig. 8 and is also used in the experiments in Section V-C. For comparison, the discrete-time norm-optimal solution [6, 4.3] is also shown in Fig. 8. Note that since the boundary effects are negligible, the performance is almost identical to that of the stable inversion approach [6, Section 4.2 and 4.3 ] and hence also inferior to that of the proposed approach.

In summary, the simulations show that the intersample performance of the special case of stable inversion is poor and that the performance of the special case of multirate inversion is moderate. Most importantly, the proposed approach offers a variety of options that outperform the two special cases and achieves superior performance. In the next section, the solution that yields the best performance in simulations is validated in experiments.

\section{Experimental validation}

In this section, the three solutions shown in Fig. 6 are validated in experiments by application to the motion system shown in Fig. 4.

For the purpose of intersample performance evaluation and due to hardware limitations, the error signal is measured with

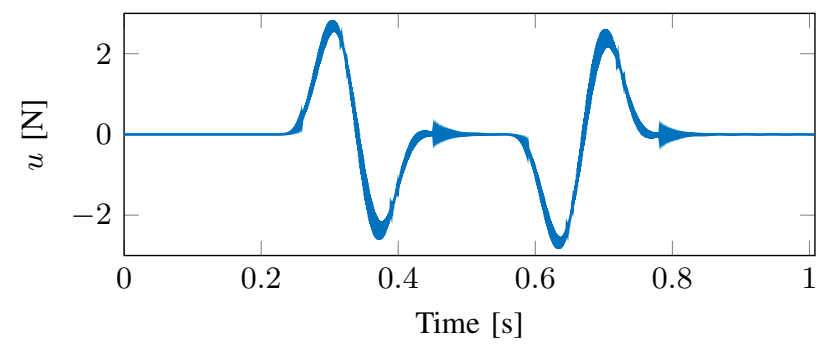

(a) Stable inversion approach.

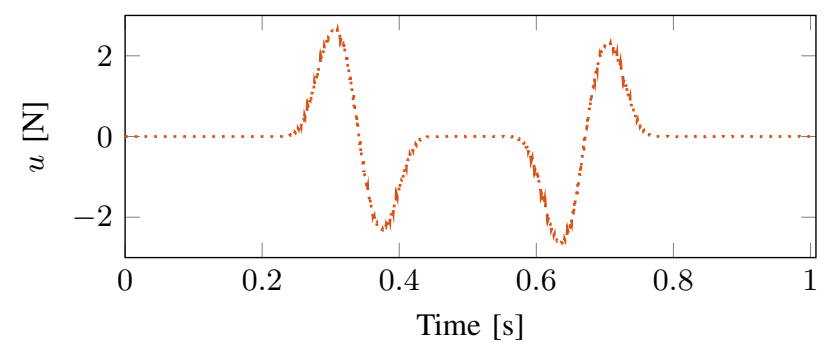

(b) Multirate inversion approach.

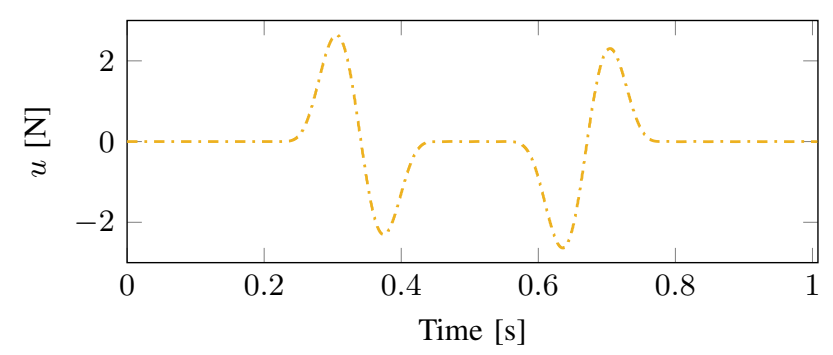

(c) Proposed approach.

Fig. 6. Generated input signals $u[k]$. The input signal for stable inversion is non smooth, whereas the input signals of multirate inversion and the proposed approach are smooth.

a sampling frequency of $12.5 \mathrm{kHz}$, i.e., 5 times larger than $f_{b}$. Besides the feedforward controllers running at the nonequidistant rate, a feedback controller running at an equidistant rate with frequency $f_{b}$ is used for stabilization. The PID controller with notch filter yields a bandwidth of $10 \mathrm{~Hz}$ and a modulus margin of $6 \mathrm{~dB}$. Due to the low bandwidth of the controller, the feedback controller has limited effect on the performance.

The experimental results are shown in Fig. 9. The results show that the proposed approach outperforms the special cases of stable inversion and multirate inversion in terms of the continuous-time error $e(t)$. Note that due to experimental conditions, e.g., model mismatches, there is no exact onsample tracking every few samples as is the case for the simulations in Section V-B.

In summary, the experiments show the practical applicability of the proposed approach. In particular, the proposed approach outperforms the special cases of stable inversion, multirate inversion as well as norm-optimal control.

\section{CONCLUSION}

A discrete-time inversion approach is developed that allows to balance the on-sample and intersample behavior for the purpose of continuous-time performance. The approach is 


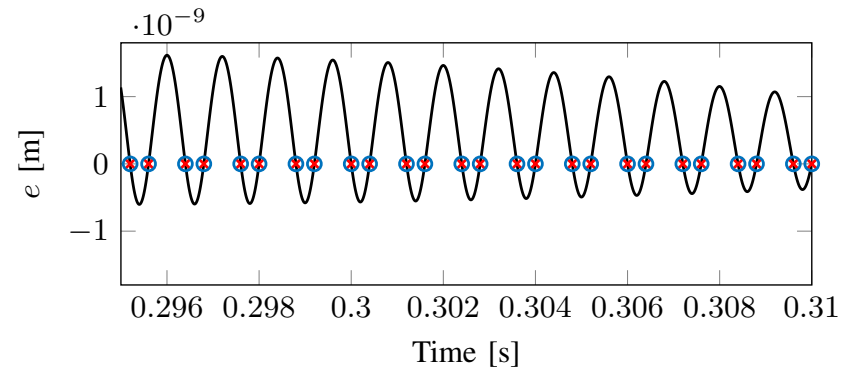

(a) The stable inversion approach achieves exact output tracking every sample (O)

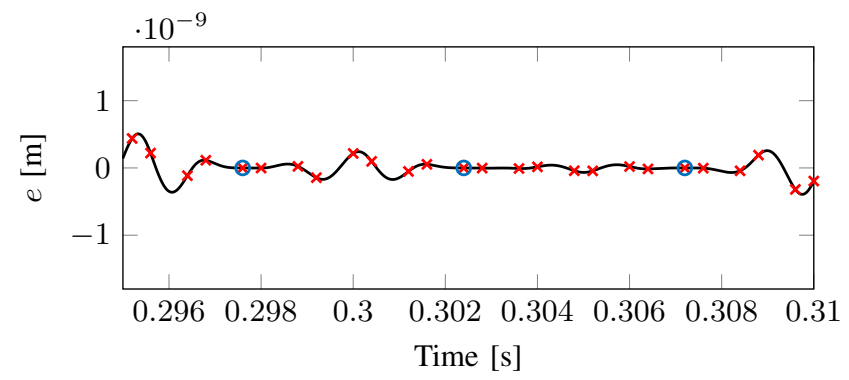

(b) The multirate inversion approach achieves exact state and output tracking every $n=8$ samples (O).

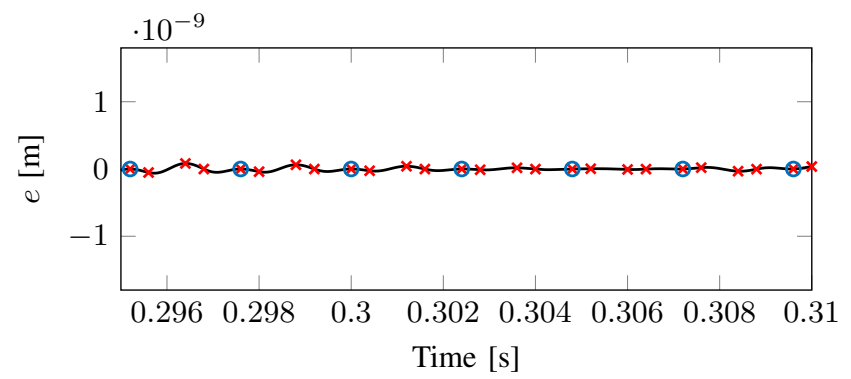

(c) The proposed approach achieves output tracking every $n_{1}=4$ samples (O).

Fig. 7. Error signals over time. The intersample error signal $e(t)(-)$ and on-sample error signal $e[k](\boldsymbol{X})$ near $t=0.30 \mathrm{~s}$ show that the proposed approach outperforms the other approaches.

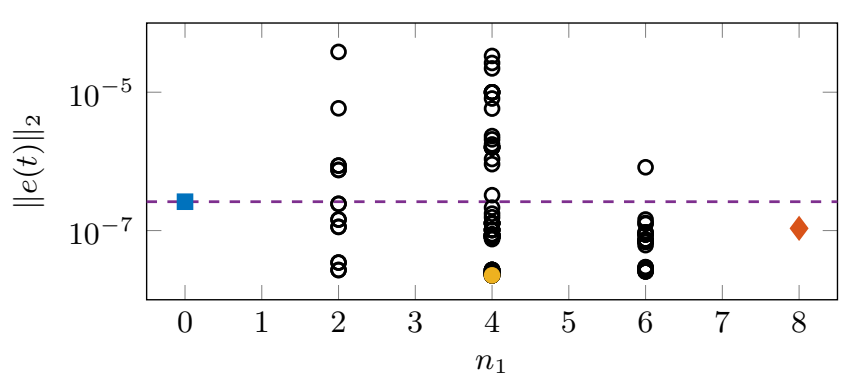

Fig. 8. Quantification of Fig. 3 through simulation. The number of states $n_{1}$ in $\underline{H}_{1}$ corresponds to the number of samples between exact on-sample tracking. The approach offers a variety of solutions $(\mathbf{O})$. The best choice for the approach $\left(n_{1}=4, \ominus\right)$ which is also shown in Fig. 6 and Fig. 7, outperforms the stable inversion approach $\left(n_{1}=0, \square\right)$ and the multirate inversion approach $\left(n_{1}=8, \diamond\right)$. For comparison, the discrete-time norm-optimal solution (- - -) is also shown and is almost identical to that of the stable inversion solution

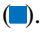

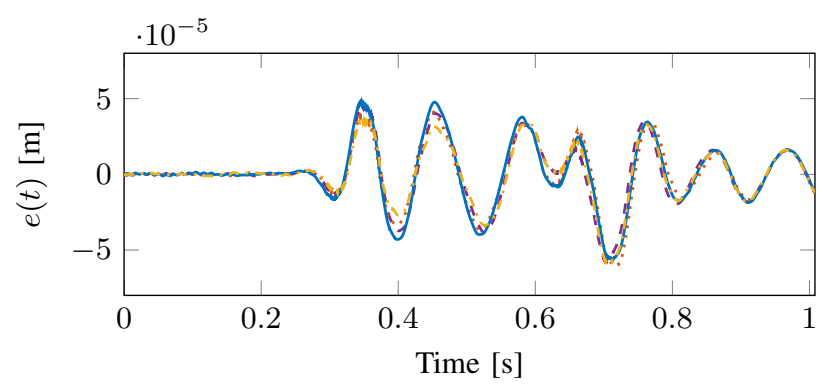

(a) Time-domain response.

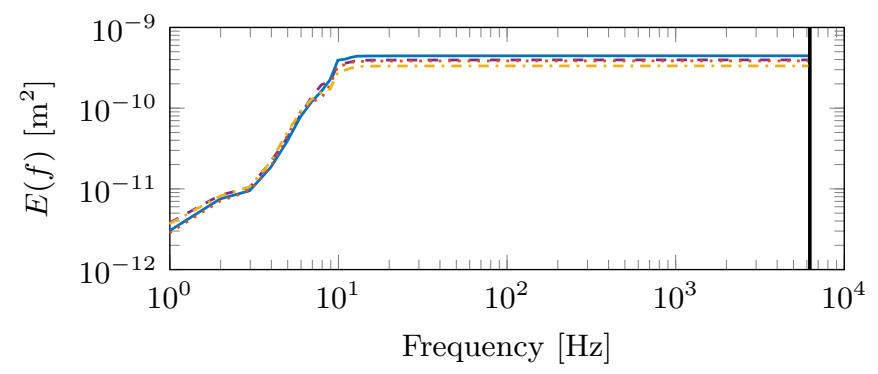

(b) Cumulative power spectrum.

Fig. 9. Experimental results showing the error signal $e(t)$ in the time and frequency domain for stable inversion $(-)$, multirate inversion $(\cdots \cdots)$, the discrete-time norm-optimal solution (- - ), and the proposed approach (---). The proposed approach outperforms the special cases of stable inversion and multirate inversion as well as the norm-optimal solution.

applicable to both LTI and LPTV systems. The multirate inversion and stable inversion approaches are recovered as special cases. Application to an LPTV motion system in both simulations and experiments demonstrates the advantages of the approach.

For LPTV systems, the approach currently involves an additional lifting step, which limits applicability due to constraints on the input and state dimensions, i.e., the state dimension should be an integer multiple of the input dimension. In contrast, stable inversion and multirate inversion can be directly applied to LPTV systems. Future work focuses on an explicit state-space decomposition for LPTV systems to avoid the additional lifting step and thereby potentially increase the performance of the approach.

Obviously, the interest is also in determining the optimal decomposition into $H_{1}$ and $H_{2}$ a priori in order to avoid exhaustive simulations. Preliminary results show that the best performance is obtained when capturing sampling zeros, introduced by zero-order-hold discretization, in $H_{1}$, and damped (anti)resonances in $\mathrm{H}_{2}$. Ongoing research focuses on more detailed guidelines in finding the optimal decomposition.

\section{APPENDIX A \\ PROOF OF THEOREM 4}

Due to the modal form of $H_{\text {mod }}$, the $A$ matrix of $H_{\text {mod }}$ is block diagonal and the states are decoupled per mode. The matrix $T_{p e r}$ is a permutation matrix and follows directly from $V, V_{\times}$and the state ordering of $H_{\text {mod }}$. The last $n_{2}$ states in (12) are uncontrollable and are redundant since the states are decoupled. Similarly, the first $n_{1}$ states in (13) are unobservable and are redundant since the states are decoupled. Hence, 
$H_{1}, H_{2}$ are minimal realizations such that $H_{\text {mod }}=H_{1} H_{2}$ in terms of transfer functions. The product $H_{1} H_{2}$ with $H_{1}, H_{2}$ in (14) is given by

$$
H_{1} H_{2}=\left[\begin{array}{cc|c}
A_{1} & B_{1} C_{2} & B_{1} D \\
0 & A_{2} & B_{2} \\
\hline C_{1} & C_{2} & D
\end{array}\right] .
$$

Using (15),

$$
\begin{aligned}
& \mathcal{T}\left(H_{1} H_{2}, T_{12}\right) \\
& =\left[\begin{array}{cc|c}
A_{1} & -A_{1} X+B_{1} C_{2}+X A_{2} & B_{1} D+X B_{2} \\
0 & A_{2} & B_{2} \\
\hline C_{1} & -C_{1} X+C_{2} & D
\end{array}\right] \\
& =\left[\begin{array}{cc|c}
A_{1} & 0 & B_{2 r} \\
0 & A_{2} & B_{2} \\
\hline C_{1} & C_{1 r} & D
\end{array}\right] .
\end{aligned}
$$

By definition of $T_{p e r}$ in (12) and (13), $\mathcal{T}\left(H_{1} H_{2}, T_{p e r}^{-1} T_{12}\right)=$ $\mathcal{T}\left(\mathcal{T}\left(H_{1} H_{2}, T_{12}\right), T_{\text {per }}^{-1}\right)=(A, B, C, D)=H_{\text {mod }}$ which concludes the proof.

\section{APPENDIX B \\ EXAMPLE OF PROCEDURE 7}

This appendix demonstrates the steps in Procedure 7 for a simple motion system.

Consider a mass-damper-spring system of which the dynamics are given by

$$
m \ddot{q}(t)+d \dot{q}(t)+k q(t)=u(t),
$$

with $q(t)$ the displacement, $\dot{q}(t)$ the velocity, $\ddot{q}(t)$ the acceleration, and the parameters mass $m=2 \mathrm{~kg}$, damping constant $d=6 \mathrm{Ns} / \mathrm{m}$, and spring constant $k=4 \mathrm{~N} / \mathrm{m}$. The reference trajectory is given by $r(t)=\sin (t)$ and the sample time is $\delta=1 \mathrm{~ms}$. For state $x(t)=\left[\begin{array}{l}\dot{q}(t) \\ q(t)\end{array}\right]$, i.e., $n=2$, the state-space realization in (1) is given by

$$
A_{c}=\left[\begin{array}{cc}
-\frac{d}{m} & -\frac{k}{m} \\
1 & 0
\end{array}\right], \quad B_{c}=\left[\begin{array}{l}
1 \\
0
\end{array}\right], \quad C_{c}=\left[\begin{array}{ll}
0 & 1
\end{array}\right] .
$$

Next, the steps in Procedure 7 are followed.

1) By Lemma 1 follows

$$
H_{c}=C_{c}\left(s I-A_{c}\right)^{-1} B_{c}=\frac{B(s)}{A(s)},
$$

with

$$
\begin{aligned}
& B(s)=\frac{\frac{1}{m}}{\frac{1}{m}}=1, \\
& A(s)=\frac{s^{2}+\frac{d}{m} s+\frac{k}{m}}{\frac{1}{m}},
\end{aligned}
$$

i.e., $b_{0}=\frac{1}{m}, a_{0}=\frac{k}{m}, a_{1}=\frac{d}{m}$. Hence, the controllable canonical form in (5) is given by

$$
H_{c c f}=\mathcal{T}\left(H_{c}, T_{c c f}\right) \stackrel{s}{=}\left[\begin{array}{cc|c}
0 & 1 & 0 \\
-\frac{d}{m} & -\frac{k}{m} & \frac{1}{m} \\
\hline 1 & 0 &
\end{array}\right],
$$

with $T_{c c f}^{-1}=\left[\begin{array}{ll}0 & 1 \\ 1 & 0\end{array}\right]$
2) Since $B(s)=1$, it follows from Theorem 2 that $\hat{x}_{c c f, s}(t)=\left[\begin{array}{l}\sin (t) \\ \cos (t)\end{array}\right]$ and $\hat{x}_{c c f, u}(t)=\left[\begin{array}{l}0 \\ 0\end{array}\right]$, hence the desired state in (9) is given by

$$
\hat{x}(t)=\left[\begin{array}{l}
\cos (t) \\
\sin (t)
\end{array}\right] .
$$

Indeed, $y(t)=C_{c} \hat{x}(t)=\sin (t)=r(t)$ and $\dot{y}(t)=$ $\cos (t)=\dot{r}(t)$.

3) The desired state of $H$ is $\hat{x}[k]=\hat{x}(k \delta)$.

4) The modal form in Theorem 4 is obtained by $H_{m o d}=$ $\mathcal{T}\left(H, T_{\text {mod }}\right)$, with

$$
T_{\text {mod }}=\left[\begin{array}{ll}
-2.8284 & -2.8284 \\
-2.2361 & -4.4721
\end{array}\right] .
$$

The desired state of $H_{m o d}$ is obtained by $\hat{x}_{\text {mod }}[k]=$ $T_{\text {mod }} \hat{x}[k]$.

5) The state-space decomposition in Theorem 4 requires a nonsingular $D$, hence a bilinear transformation is used, in particular

$$
\begin{aligned}
\tilde{H}_{\text {mod }} & =\mathcal{B}\left(H_{\text {mod }}\right) \\
& \stackrel{s}{=}\left[\begin{array}{cc|c}
0.001 & 0 & -0.001 \\
0 & -0.0005 & -0.00079 \\
\hline 0.2502 & -0.3164 & -6.25 \times 10^{-11}
\end{array}\right] .
\end{aligned}
$$

The transfer function decomposition of $\tilde{H}_{\text {mod }}$ is obtained through Lemma 3 by selecting the column spaces of the invariant subspaces as $\tilde{V}=\left[\begin{array}{l}1 \\ 0\end{array}\right]$ and $\tilde{V}_{\times}=\left[\begin{array}{l}-0.7845 \\ -0.6202\end{array}\right]$, such that $\tilde{\Pi}=\left[\begin{array}{cc}1 & -1.2649 \\ 0 & 0\end{array}\right]$, see also (10), which yields

$$
\begin{aligned}
& \tilde{H}_{1 f} \stackrel{s}{=} {\left[\begin{array}{cc|c}
0.001 & 0 & -4 \\
0 & -0.0005 & 0 \\
\hline 0.2502 & -0.3164 & 1
\end{array}\right], } \\
& \tilde{H}_{2 f} \stackrel{s}{=}\left[\begin{array}{cc|c}
0.001 & 0 & -0.001 \\
0 & -0.0005 & -0.00079 \\
\hline 0 & 0.00016 & -6.25 \times 10^{-11}
\end{array}\right] .
\end{aligned}
$$

By selecting $T_{p e r}=\left[\begin{array}{ll}1 & 0 \\ 0 & 1\end{array}\right],(12)$ and (13) are satisfied and the minimal state-space realizations $\tilde{H}_{1}$ and $\tilde{H}_{2}$ are obtained. Undoing the bilinear transformation yields

$$
\begin{aligned}
& H_{1}=\mathcal{B}^{-1}\left(\tilde{H}_{1}\right) \stackrel{z}{=}\left[\begin{array}{c|c}
0.998 & -5.651 \\
\hline 0.3536 & 0
\end{array}\right] \\
& H_{2}=\mathcal{B}^{-1}\left(\tilde{H}_{2}\right) \stackrel{z}{=}\left[\begin{array}{c|c}
0.999 & -0.001117 \\
\hline 0.0002235 & -1.25 \times 10^{-7}
\end{array}\right] .
\end{aligned}
$$

System $H_{12}$ follows from (23).

6) The desired state of $H_{12}$ is given by $\hat{x}_{12}[k]=$ $T_{12} T_{\text {per }} \hat{x}_{\text {mod }}[k]$, with $T_{12}$ in (16) given by

$$
T_{12}=\left[\begin{array}{cc}
1 & 1.2649 \\
0 & 1
\end{array}\right]
$$

with $X=1.2649$ the solution to the Sylvester equation (15). 
7) The desired state for $H_{1}$ is given by $\hat{x}_{1}[k]=$

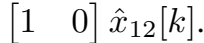

This concludes the application of Procedure 7 to the system in (32).

\section{REFERENCES}

[1] S. A. Rios, A. J. Fleming, and Y. K. Yong, "Monolithic Piezoelectric Insect With Resonance Walking," IEEE/ASME Transactions on Mechatronics, vol. 23, no. 2, pp. 524-530, 2018.

[2] L. Blanken, F. Boeren, D. Bruijnen, and T. Oomen, "Batch-to-Batch Rational Feedforward Control: From Iterative Learning to Identification Approaches, With Application to a Wafer Stage," IEEE/ASME Transactions on Mechatronics, vol. 22, no. 2, pp. 826-837, 2017.

[3] Z. Li and J. Shan, "Modeling and Inverse Compensation for Coupled Hysteresis in Piezo-Actuated Fabry-Perot Spectrometer," IEEE/ASME Transactions on Mechatronics, vol. 22, no. 4, pp. 1903-1913, 2017.

[4] S. Devasia, D. Chen, and B. Paden, "Nonlinear Inversion-Based Output Tracking," IEEE Transactions on Automatic Control, vol. 41, no. 7, pp. 930-942, 1996.

[5] T. Chen and B. A. Francis, Optimal Sampled-Data Control Systems. London, Great Britain: Springer, 1995.

[6] J. van Zundert and T. Oomen, "On inversion-based approaches for feedforward and ILC," IFAC Mechatronics, vol. 50, pp. 282-291, 2018

[7] M. Tomizuka, "Zero Phase Error Tracking Algorithm for Digital Control," Journal of Dynamic Systems, Measurement, and Control, vol. 109, no. 1, pp. 65-68, 1987.

[8] E. Gross, M. Tomizuka, and W. Messner, "Cancellation of Discrete Time Unstable Zeros by Feedforward Control," Journal of Dynamic Systems, Measurement, and Control, vol. 116, no. 1, pp. 33-38, 1994.

[9] K. L. Moore, S. Bhattacharyya, and M. Dahleh, "Capabilities and Limitations of Multirate Control Schemes," Automatica, vol. 29, no. 4, pp. 941-951, 1993.

[10] T. Oomen, J. van de Wijdeven, and O. Bosgra, "Suppressing intersample behavior in iterative learning control," Automatica, vol. 45, no. 4, pp. 981-988, 2009.

[11] W. Ohnishi, T. Beauduin, and H. Fujimoto, "Preactuated Multirate Feedforward Control for Independent Stable Inversion of Unstable Intrinsic and Discretization Zeros," IEEE/ASME Transactions on Mechatronics, 2019.

[12] H. Fujimoto, Y. Hori, and A. Kawamura, "Perfect Tracking Control Based on Multirate Feedforward Control with Generalized Sampling Periods," IEEE Transactions on Industrial Electronics, vol. 48, no. 3 , pp. 636-644, 2001.

[13] H. Fujimoto and Y. Hori, "High-performance servo systems based on multirate sampling control," Control Engineering Practice, vol. 10, no. 7, pp. 773-781, 2002.

[14] J. van Zundert, J. Bolder, S. Koekebakker, and T. Oomen, "Resourceefficient ILC for LTI/LTV systems through LQ tracking and stable inversion: Enabling large feedforward tasks on a position-dependent printer," IFAC Mechatronics, vol. 38, pp. 76-90, 2016.

[15] J. van Zundert and T. Oomen, "LPTV Loop-Shaping with Application to Non-Equidistantly Sampled Precision Mechatronics," in Proceedings of the 15th International Workshop on Advanced Motion Control, Tokyo, Japan, 2018, pp. 467-472.

[16] Y. Yamamoto, "A Function Space Approach to Sampled Data Control Systems and Tracking Problems," IEEE Transactions on Automatic Control, vol. 39, no. 4, pp. 703-713, 1994

[17] B. A. Bamieh and J. B. Pearson Jr., "A General Framework for Linear Periodic Systems with Applications to $H^{\infty}$ Sampled-Data Control," IEEE Transactions on Automatic Control, vol. 37, no. 4, pp. 418-435, 1992.

[18] G. C. Goodwin, S. F. Graebe, and M. E. Salgado, Control System Design. Upper Saddle River, New Jersey, 2000

[19] A. V. Oppenheim, A. S. Willsky, and S. H. Nawab, Signals and Systems, 2nd ed. Prentice-Hall, Inc., 1997.

[20] S. Devasia, "Output Tracking with Nonhyperbolic and Near Nonhyperbolic Internal Dynamics: Helicopter Hover Control," in Proceedings of the 1997 American Control Conference, Albuquerque, New Mexico, 1997, pp. 1439-1446.

[21] H. Bart, I. Gohberg, M. Kaashoek, and A. Ran, "Schur complements and state space realizations," Linear Algebra and its Applications, vol. 399, pp. 203-224, 2005.

[22] G. F. Franklin, J. D. Powell, and A. Emami-Naeini, Feedback Control of Dynamic Systems, 7th ed. Upper Saddle River, New Jersey: Pearson, 2015.
[23] R. Bartels and G. Stewart, "Solution of the Matrix Equation AX + XB $=\mathrm{C}[\mathrm{F} 4]$," Communications of the ACM, vol. 15, no. 9, pp. 820-826, 1972.

[24] J. van Zundert and T. Oomen, "Stable inversion of LPTV systems with application in position-dependent and non-equidistantly sampled systems," International Journal of Control, vol. 92, no. 5, pp. 10221032, 2019.

[25] A. Hara, K. Saiki, K. Sakata, and H. Fujimoto, "Basic Examination on Simultaneous Optimization of Mechanism and Control for High Precision Single Axis Stage and Experimental Verification," in Proceedings of the 34th Annual Conference of Industrial Electronics, Orlando, Florida, 2008, pp. 2509-2514.

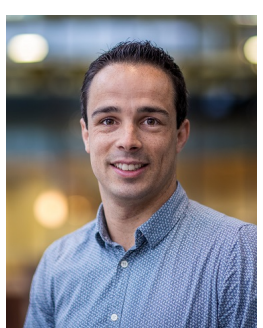

Jurgen van Zundert received the M.Sc. degree (with great appreciation) in Mechanical Engineering from the Eindhoven University of Technology, Eindhoven, The Netherlands in 2014.

$\mathrm{He}$ is currently pursuing the Ph.D. degree in the Control Systems Technology group within the department of Mechanical Engineering at TU/e.

His research interests are in feedforward motion control, multi-rate control, and iterative learning control.

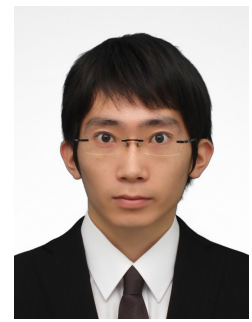

Wataru Ohnishi received the B.E., M.S., and Ph.D degrees from the University of Tokyo, Japan in 2013, 2015, and 2018, respectively.

Presently, he is a research associate with the Department of Electrical Engineering and Information Systems, Graduate School of Engineering, the University of Tokyo.

His research interest includes high-precision motion control. He is a member of the Institute of Electrical Engineers of Japan.

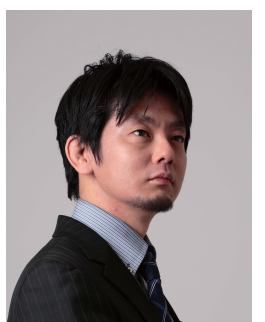

Hiroshi Fujimoto (S'99-M'01-SM'12) received his $\mathrm{Ph} . \mathrm{D}$. degree from the the Department of Electrical Engineering, the University of Tokyo, in 2001. In 2001, he joined the Department of Electrical Engineering, Nagaoka University of Technology, Niigata, Japan, as a research associate. From 2002 to 2003 , he was a visiting scholar at the School of Mechanical Engineering, Purdue University, U.S.A. In 2004, he joined the Department of Electrical and Computer Engineering, Yokohama National University, Yokohama, Japan, as a lecturer and went on to become an associate professor in 2005 . He is currently an associate professor at the University of Tokyo since 2010.

He received the Best Paper Awards from the IEEE Transactions on Industrial Electronics in 2001 and 2013, Isao Takahashi Power Electronics Award in 2010, Best Author Prize of SICE in 2010, The Nagamori Grand Award in 2016, and First Prize Paper Award IEEE Transactions on Power Electonics in 2016.

His interests are in control engineering, motion control, nano-scale servo systems, electric vehicle control, and motor drives. Dr. Fujimoto is a member of the Society of Instrument and Control Engineers, the Robotics Society of Japan, and the Society of Automotive Engineers of Japan, as well as a senior member of IEE of Japan. 


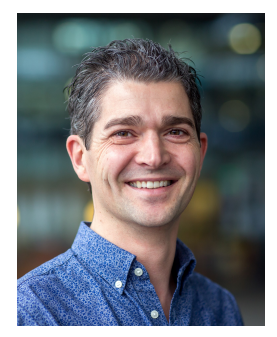

Tom Oomen received the M.Sc. degree (cum laude) and $\mathrm{Ph} . \mathrm{D}$. degree from the Eindhoven University of Technology, Eindhoven, The Netherlands.

$\mathrm{He}$ held visiting positions at KTH, Stockholm, Sweden, and at The University of Newcastle, Australia. Presently, he is associate professor with the Department of Mechanical Engineering at the Eindhoven University of Technology. He is a recipient of the Corus Young Talent Graduation Award, the 2015

IEEE Transactions on Control Systems Technology Outstanding Paper Award, the 2017 IFAC Mechatronics Best Paper Award, and recipient of a Veni and Vidi personal grant. $\mathrm{He}$ is Associate Editor of the IEEE Control Systems Letters (L-CSS), IFAC Mechatronics, and IEEE Transactions on Control Systems Technology. He is a member of the Eindhoven Young Academy of Engineering.

His research interests are in the field of data-driven modeling, learning, and control, with applications in precision mechatronics. 